those treated with LTA (6.5 years vs 4.4 years), as was 5-year overall survival (53.4\% vs $38.9 \%$ ). Patients who received LTA also had more complications than those who received TH. The probability of LTA being significantly superior to $\mathrm{TH}$ was predicted to be just $3.65 \%$, and as a result recruitment of patients was terminated in December 2003. Updated survival data in March 2006 showed a median overall survival of 5.7 years for patients assigned $\mathrm{TH}$, and 3.5 years for those assigned LTA; 5-year overall survival was $52.3 \%$ and $37.9 \%$, respectively.

The authors conclude that use of LTA cannot be justified in the treatment of Siewert type II-III adenocarcinoma of the gastric body or cardia.

Original article Sasako M et al. (2006) Left thoracoabdominal approach versus abdominal-transhiatal approach for gastric cancer of the cardia or subcardia: a randomised controlled trial. Lancet Oncol 7: 644-651

\section{Micrometastases predict relapse and survival in high-risk breast cancer}

A recent study has demonstrated that the presence of circulating micrometastases, detected using real-time polymerase chain reaction assays for a panel of breast cancer-specific mRNA transcripts, predicts relapse and death in patients with high-risk breast cancer (HRBC) undergoing high-dose chemotherapy after receiving adjuvant chemotherapy.

Peripheral blood cells were collected by apheresis several months after surgery from 84 patients with HRBC who had received adjuvant chemotherapy (fluorouracil, epirubicin, and cyclophosphamide for six cycles, or doxorubicin and cyclophosphamide followed by paclitaxel) before undergoing one course of cyclophosphamide, thiotepa and carboplatin (STAMP V). Data on the presence of peripheral blood micrometastases detected by real-time polymerase chain reaction, together with a range of tumor-related and treatment-related parameters, were analyzed.

At a median follow-up of 68.3 months, 47 patients (56\%) had relapsed; 35 deaths $(41.7 \%)$ had been registered by the end of the study. In Cox multivariate analysis, tumor size and presence of micrometastases were significantly associated with relapse $(P=0.0470$ and $P=0.006$, respectively); for patients with peripheral blood micrometastases, hazard ratios were $269 \%$ for relapse $(P=0.006)$ and $300 \%$ for death $(P=0.011)$. Patients with micrometastases had a significantly shorter time to relapse than patients without micrometastases (31.3 vs 84.2 months; $P=0.021$ ).

Micrometastases after adjuvant chemotherapy are, therefore, strong predictors of relapse and death in $\mathrm{HRBC}$ patients undergoing high-dose chemotherapy. The use of a gene panel rather than only one or two genes to identify micrometastases seems to increase accuracy.

Original article Quintela-Fandino M et al. (2006) Breast cancer-specific mRNA transcripts presence in peripheral blood after adjuvant chemotherapy predicts poor survival among high-risk breast cancer patients treated with highdose chemotherapy with peripheral blood stem cell support. JClin Oncol 24: 3611-3618

\section{Correlation between physical activity, colorectal cancer and survival}

Regular physical activity is associated with a reduced risk of colon/colorectal cancer; two articles published concurrently in the Journal of Clinical Oncology report that exercise could also be beneficial after diagnosis of disease.

In the first paper, the impact of physical activity on colorectal-cancer-specific survival and overall mortality was studied in 573 women with stage I-III colorectal cancer from the prospective Nurses' Health Study. Only women who survived more than 6 months after their postdiagnosis assessment were included in analyses. Physical activity was measured using metabolic equivalent task (MET) hours per week. Over a median 9.6-year follow-up, both cancer-specific and overall mortality levels were associated with amount of exercise after diagnosis. Women who did $\geq 18$ MET-hours of physical activity a week following diagnosis had adjusted hazard ratios (HRs) of 0.39 for colorectal-cancer-specific mortality and 0.43 for overall mortality compared with those who did <3 MET-hours/week. Exclusion of patients who died within 12 and 24 months of assessment did not affect the results. Although prediagnosis level of activity did not predict type of mortality, women who increased their activity levels following 